\title{
La historiografía del patrimonio educativo en España: un balance crítico ${ }^{1}$
}

\section{A historiografia do patrimônio educativo na Espanha: um balanço crítico}

\section{Educational heritage historiography in Spain: a critical balance}

\author{
Pedro L. Moreno Martínez²
}

\begin{abstract}
RESUMEN
La historiografía del patrimonio educativo en España ha experimentado, a lo largo de los últimos quince años, avances significativos. En este estudio se efectúa una aproximación al análisis de algunas de las vías principales que más han contribuido a impulsar la renovación y la difusión de la historiografía del patrimonio educativo en nuestro país. En primer lugar se aborda el papel desempeñado por las exposiciones pedagógicas como instrumento al servicio de la promoción de la investigación y su divulgación. En segundo lugar, se presenta el despegue alcanzado por el museísmo pedagógico y las vinculaciones existentes entre ciertos tipos de museos, los grupos de investigación universitarios existentes y los proyectos de investigación desarrollados. Por último, se analiza el papel desempañado por las reuniones científicas en el avance del conocimiento tomando como referencia las aportaciones efectuadas a las jornadas científicas de la Sociedad Española para el Estudio del Patrimonio Histórico-Educativo (SEPHE).
\end{abstract}

Palabras clave: historiografía; patrimonio educativo; museísmo pedagógico; jornadas científicas; España.

DOI: $10.1590 / 0104-4060.43468$

1 Este artículo se ha llevado a cabo en el marco del proyecto de investigación titulado "Imagen y educación: marketing, comercialización, didáctica (España, siglo XX)”, EDU2013-42040-P, financiado por el Ministerio de Economía y Competitividad del Gobierno de España en el Plan Estatal de Investigación Científica y Técnica y de Innovación 2013-2016.

2 Universidad de Murcia. Murcia, España. Avda. Teniente Flomesta, 5 - 30003. E-mail: plmoreno@um.es 


\title{
RESUMO
}

A historiografia do patrimônio educativo na Espanha tem experimentado, ao longo dos últimos quinze anos, avanços significativos. Neste estudo realiza-se uma aproximação das análises de algumas das linhas principais que têm contribuído para a renovação e difusão da historiografia do patrimônio educativo na Espanha. Em primeiro lugar aborda-se o papel desempenhado pelas exposições pedagógicas como instrumento a serviço da promoção da investigação e sua divulgação. Em segundo lugar, apresenta-se o desenvolvimento alcançado pela musealização pedagógica e as vinculações existentes entre certos tipos de museus, os grupos de pesquisa universitários existentes e os projetos de investigação desenvolvidos. Por último, analisa-se o papel desempenhado pelos encontros científicos no avanço do conhecimento, tomando como referência os apontamentos realizados nas jornadas científicas da Sociedade Espanhola para o Estudo do Patrimônio Histórico-Educativo (SEPHE).

Palavras-chave: historiografia; patrimônio educativo; musealização pedagógica; jornadas científicas; Espanha.

\begin{abstract}
Educational heritage historiography in Spain has experienced significant improvement in the last fifteen years. In this study an approximation of analyses on some of the main lines which have contributed to the renewal and dissemination of educational heritage historiography in Spain takes place. Firstly, the role played by pedagogical exhibitions was approached, as an instrument at the service of promoting their investigation and spreading. Secondly, the development reached by educational musealization and the existing bonds among some types of museums, the existing university research groups and the investigation projects developed are presented. Finally, the role played by the scientific meetings in knowledge improvement is studied, taking as reference the observations from the scientific journeys of the Spanish Society for the Historical-Educational Heritage Study (SEPHE).

Keywords: historiography; educational heritage; pedagogical musealization; scientific journeys; Spain.
\end{abstract}

\section{Introducción}

La investigación histórica sobre el patrimonio educativo ha alcanzado progresivamente, a lo largo de las dos últimas décadas, una atención destacada 
en el contexto internacional. A ello han contribuido, entre otros factores, las corrientes de renovación historiográfica, impulsadas por propuestas como la enunciada por el historiador Dominique Julia en 1995, de considerar la cultura escolar como objeto histórico; los avances asociados a la nueva historia cultural de la educación, a través de sus líneas etnográfica y microhistórica; los movimientos de recuperación de la memoria y el debatido problema de las relaciones entre memoria e historia; las declaraciones y las recomendaciones internacionales que han contribuido a impulsar políticas de protección, conservación y difusión del patrimonio y a ampliar las categorías de bienes susceptibles de ser salvaguardados, etc.

Nuestro país no ha permanecido ajeno a estas tendencias historiográficas, produciéndose un notable y creciente interés académico y social por el patrimonio educativo especialmente reseñable durante los últimos quince años, como se constata, entre otros elementos, en la proliferación de exposiciones pedagógicas, en la recuperación y el desarrollo experimentado por el museísmo pedagógico; el impulso alcanzado en el ámbito académico con la gestación de grupos de investigación en las universidades españolas, el desarrollo de proyectos de investigación y publicaciones; la constitución de sociedades científicas o redes nacionales e internacionales, o la promoción de encuentros, seminarios, reuniones técnicas y congresos científicos nacionales e internacionales. En este artículo analizaremos algunas de las vías principales que han contribuido a la renovación y difusión de la historiografía del patrimonio educativo en España.

\section{El furor de las exposiciones pedagógicas}

Como lamentaba Martin Lawn (2009, p. 7), los historiadores de la educación nunca han prestado la debida atención al estudio del papel desempeñado por las exposiciones pedagógicas, como las realizadas con motivo de la celebración de las Exposiciones Universales, en la conformación y modernización de la educación. Mucho nos queda por conocer acerca de tales eventos y la presencia que los estados y las florecientes industrias escolares tuvieron en los mismos desde sus inicios, a partir de la segunda mitad del siglo XIX, hasta nuestros días. (HERNÁNDEZ DÍAZ, 1993; LAWN, 2009; POZO ANDRÉS, 2009). Las exposiciones pedagógicas nacionales e internacionales gestadas con el propósito de mostrar los avances tecnológicos o industriales de material de enseñanza, en congresos o en las referidas Exposiciones Universales, a lo largo de la segunda mitad del siglo XIX, terminarían transformándose en exposiciones permanen- 
tes dando lugar a la creación de los museos pedagógicos decimonónicos. En la actualidad, al menos en nuestro país, el auge alcanzado por las exposiciones pedagógicas también parece guardar cierta relación tanto con el interés creciente por el patrimonio educativo así como, veremos más adelante, con los avances mostrados por el museísmo pedagógico coetáneo.

Tras la restauración democrática en España, a partir de 1978, en un nuevo momento histórico, no ajeno a cierto activismo político, cultural y a los movimientos de recuperación de la memoria, las exposiciones han sido uno de los medios para rescatar del olvido y reconstruir la memoria individual, social e institucional. Una somera aproximación a las mismas permite constatar la proliferación de la organización de exposiciones pedagógicas a lo largo de las tres últimas décadas. Durante los últimos años, sólo las páginas del Boletín Informativo SEPHE. Sociedad Española para el Estudio del Patrimonio Histórico-Educativo, de los números anuales publicados de enero de 2006 a enero de 2014, reseñaban la celebración de 72 exposiciones. (BOLETÍN INFORMATIVO SEPHE, 2006-2014).

El espectro temático de las exposiciones realizadas ha sido muy diverso. Entre aquellos temas que parecen haber aglutinado o inspirado un mayor número de exposiciones podemos mencionar un primer grupo dedicado al profesorado, a conmemorar a personalidades relevantes del pensamiento y la educación, como, por ejemplo, Lorenzo Luzuriaga, Manuel Bartolomé Cossío, Leopoldo Alas Clarín, Miguel de Unamuno, Francisco Ferrer i Guardia o Benito Pérez Galdós, colectivos docentes, o maestros singulares destacados, como Félix Martí Alpera, Aurelio Rodríguez Charentón, etc. Un segundo grupo constituido por un abundante número de exposiciones destinadas a conmemorar la gestación o trayectoria de una institución educativa determinada. Las más numerosas han sido aquellas preparadas por las antiguas Escuelas Normales, dedicadas a la formación de maestros y maestras, llevadas a cabo fundamentalmente durante la década de los noventa, para celebrar la conmemoración del 150 aniversario, ya que gran parte de ellas fueron fundadas durante los años cuarenta del siglo XIX, o el 150 aniversario, igualmente, de los Institutos Provinciales de Segunda Enseñanza surgidos a partir del Plan Pidal de 1845, así como de exposiciones temáticas de los valiosos fondos científicos, bibliográficos y documentales conservados en tales instituciones. También cabría mencionar, en este grupo, aquellas muestras de carácter conmemorativo promovidas por universidades, centros públicos singulares o privados pertenecientes a congregaciones religiosas, o por instituciones tan relevantes como el inicialmente denominado Ministerio de Instrucción Pública y Bellas Artes o la Junta para la Ampliación de Estudios e Investigaciones Científicas, etc. Probablemente, el grupo de exposiciones más numeroso es aquel que ha tendido a recrear la trayectoria de los 
centros educativos, en especial de la escuela primaria, a lo largo de un periodo dilatado o una etapa histórica concreta, como la Segunda República, el franquismo o momentos más recientes, o que han centrado su atención en materiales de enseñanza específicos como cuadernos escolares, material científico, los dones y las ocupaciones de Froebel, etc. Un cuarto grupo temático estaría formado por aquellas exposiciones sobre editoriales significadas, como Hernando o Calleja, la manualística escolar, manuales de disciplinas o saberes específicos, libros de lectura como El Quijote, etc. Un quinto grupo englobaría las exposiciones relativas a la historia de la infancia, tales como las centradas en el mundo del juguete, la literatura infantil, los dibujos, la guerra o el exilio. Un sexto grupo temático vendría dado por aquellas que se ocupan de colectivos específicos, tales como mujeres, niñas, movimientos o asociaciones juveniles, etc. Un séptimo grupo de exposiciones destinado a conmemorar iniciativas educativas singulares como, por ejemplo, las Misiones Pedagógicas. (HERNÁNDEZ DÍAZ, 2003; BOLETÍN INFORMATIVO SEPHE, 2006-2014).

Una primera y somera aproximación al análisis de las exposiciones pedagógicas celebradas en España a lo largo de las tres últimas décadas, que requiere un tratamiento más detallado, exhaustivo y profundo de lo que posibilita un conciso apartado, permite atisbar la importancia y la proyección de las mismas por su abundancia, diversidad temática, la heterogeneidad de las instituciones que las han promovido, el empleo progresivo, entre otras modalidades, de exposiciones virtuales, etc. Entre las interrelaciones existentes entre las exposiciones y la historiografía del patrimonio habría que destacar, al menos, una doble función. Por una parte, las exposiciones han constituido una vía de estímulo e impulso de la investigación para favorecer la recuperación y reconstrucción de la memoria, la historia de la educación y su patrimonio, y por otra, un resultado, un medio de transferencia y difusión de la investigación desarrollada, reflejada en los catálogos publicados y en su contribución a dotar de mayor visibilidad y proyección académica y social al patrimonio educativo.

\section{El museísmo pedagógico en España}

El despegue actual de los museos pedagógicos en España ha sido una vía fundamental para potenciar la salvaguarda y difusión del patrimonio, así como para impulsar la investigación del patrimonio educativo. La incorporación de España al museísmo pedagógico se produjo, inicialmente, en un período en el que tales instituciones comenzaron su implantación internacional durante la 
segunda mitad del siglo XIX. Unos museos que, como los de Leizpig, creado en 1865, Viena, en 1872, Zurich, en 1873, Munich, en 1875, Berlín, en 1877, París, en 1879, Bruselas, en 1880, o Dresde, en 1881, visitaría Manuel Bartolomé Cossío previamente a ser nombrado primer director del denominado Museo Pedagógico Nacional, una institución emblemática que perduraría desde su creación, en 1882, a su supresión por el régimen franquista, en 1941. (GARCÍA DEL DUJO, 1985; OTERO URTAZA, 2008).

La recuperación e impulso actual de los museos pedagógicos en España se ha producido, no sin cierto retraso, en sintonía con el proceso de revitalización y eclosión de las instituciones museísticas vinculadas al mundo de la educación experimentada en las últimas décadas en el contexto internacional, en general, y en los países europeos, en particular como, entre otros, en Alemania, Dinamarca, Francia o Reino Unido. Unos museos de los que no es fácil precisar su número, de los que solo en Europa se estima puedan existir unos cuatrocientos, de los que tres cuartas partes se han gestado o recuperado durante las tres últimas décadas. (RUIZ BERRIO, 2010, p. 133).

En España carecemos de un registro nacional que nos ofrezca un inventario inequívoco de las iniciativas existentes. Según la información publicada periódicamente en las páginas del Boletín Informativo SEPHE, desde la creación de los primeros museos a finales de la década de los ochenta hasta el presente, contamos con un total de medio centenar de museos y colecciones de desigual entidad. (BOLETÍN INFORMATIVO SEPHE, 2006-2014). Los intentos de establecer tipologías que permitan clasificar, en base a algunas de sus características, la gama de iniciativas museísticas existentes, evidencia su diversidad y heterogeneidad incrementada, durante la última década, con la incorporación de espacios museísticos virtuales. (CAMPILLO FLORES; COLLELDEMONT PUJADAS, 2007). Atendiendo a su dependencia institucional podemos hablar, entre otros, de tres tipos de entidades que queremos destacar especialmente no solo por la labor desarrollada en favor de la salvaguarda y difusión de los bienes patrimoniales, sino por su destacado papel asociado a la promoción de la investigación del patrimonio educativo.

El primero de ellos estaría formado por aquellos museos pedagógicos creados oficialmente por las Administraciones Autonómicas. Son los casos del Museo Pedagógico de Galicia (MUPEGA), fundado en 2000, el Centro de Recursos, Interpretación y Estudios de la Escuela (CRIEME) de Cantabria, inaugurado en 2005, o el Museo Pedagógico de Aragón, constituido en 2006. Instituciones que han favorecido la investigación del patrimonio educativo a través de vías tales como, por ejemplo, la organización y celebración de congresos nacionales e internacionales, la gestación de servicios de documentación, la promoción de publicaciones o la edición de publicaciones periódicas como Cabás - revista 
digital sobre Patrimonio Histórico Educativo (en: <http://revista.muesca.es $>$ ) o aragóneduca. Revista del Museo Pedagógico de Aragón (en: <http://www. museopedagogicodearagon.com/ $>$ ).

Un segundo grupo estaría compuesto por los museos pedagógicos o de historia de la educación y los centros de estudios creados por las universidades. Su nacimiento, generado, principalmente, a lo largo de la última década, ha sido impulsado por los grupos de investigación gestados en las instituciones de educación superior. La labor emprendida por estos grupos de investigación ha sido determinante para la promoción de la investigación y el avance del conocimiento sobre patrimonio educativo en nuestro país. Unos grupos de investigación que han concurrido a las convocatorias públicas promovidas por organismos internacionales, nacionales y autonómicos de investigación desarrollando un destacado conjunto de proyectos sobre facetas muy diversas del patrimonio educativo en las que han estado presentes, de forma preponderante el museísmo pedagógico, pero también la cultura material de las instituciones educativas, la manualística escolar, los usos didácticos del patrimonio, las nuevas tecnologías de la información y la comunicación y el patrimonio, el inventario y documentación del patrimonio, la imagen, la memoria de los docentes, etc.

Los museos y centros de estudios generados han sido, al unísono, una de las consecuencias de tal impulso investigador, así como una nueva plataforma al servicio de la potenciación, transferencia y difusión de la investigación. Entre los museos universitarios hay que mencionar el Museo de Historia de la Educación "Manuel Bartolomé Cossío" y el Museo Pedagógico de Arte Infantil (MUPAI), ambos de la Universidad Complutense de Madrid, el Museo de Educación de la Universidad de La Laguna, el Centro Museo Pedagógico (CEMUPE), de la Universidad de Salamanca (Zamora), el Museo Pedagógico de la Universidad de Huelva, el Museo Pedagógico de la Universidad de Sevilla, el Seminario-Museo de Historia de la Escuela de la Univeritat de València, el Museo de la Educación, de la Universidad del País Vasco (San Sebastián); museos virtuales como el Museo Universitari Virtual de Pedagogia, de Universidad de Vic (MUVIP) (Barcelona) (en: < http://www.uvic.cat/muvip>), el Museo Virtual de Historia de la Educación, de la Universidad de Murcia (MUVHE) (en: <http://www.um.es/ muvhe/user/>) o el Museo Virtual de Educación "Manuel Bartolomé Cossío", de la Universidad Complutense de Madrid (en: <http://pendientedemigracion. ucm.es/info/muscosio/>), así como centros de estudios como el Centro de Investigación MANES, de la Universidad Nacional de Educación a Distancia (UNED, Madrid), o el Centro de Estudios sobre la Memoria Educativa (CEME), de la Universidad de Murcia.

Un tercer grupo de iniciativas estaría formado por entidades museísticas promovidas por asociaciones culturales. Dentro de ellas cabe destacar, espe- 
cialmente, al Centro Internacional de la Cultura Escolar (CEINCE), promovido y dirigido, desde su inauguración en 2008, por el profesor Agustín Escolano Benito. El CEINCE, radicado en Berlanga de Duero (Soria), es una institución de referencia internacional en el estudio y difusión de la cultura de la escuela en su perspectiva histórica y actual, como queda de manifiesto en las iniciativas desarrolladas (en: <http://www.ceince.eu/>).

\section{Jornadas científicas y el avance del conocimiento}

La constitución de sociedades científicas, como la Sociedad Española para el Estudio del Patrimonio Histórico-Educativo (SEPHE) en 2004, de redes nacionales como la Asociación Nacional para la Defensa del Patrimonio de los Institutos Históricos (ANDPIH) en 2007, o internacionales, en las que también participan investigadores de nuestro país, como la Red Ibero Americana para la Investigación y Difusión del Patrimonio Histórico-Educativo (Rede Iberoamericana para a Investigacão e a Difusão do Patrimônio Histórico-Educativo) (RIDPHE) gestada en 2007 o, recientemente, la Red Internacional de Educación Patrimonial (International Network on Heritage Education) (RIEP/INHE), originada en el II Congreso Internacional de Educación Patrimonial celebrado en São Paulo en noviembre de 2014, pueden contribuir a potenciar la protección, conservación e investigación, así como la promoción, estímulo, apoyo y difusión del patrimonio educativo.

La celebración de encuentros, seminarios, reuniones técnicas y congresos científicos nacionales e internacionales ha sido una de las vías más fecundas y efectivas para impulsar y dar a conocer en nuestro país la investigación histórica acerca del patrimonio educativo. Unas reuniones científicas promovidas, entre otras instancias, por la Sociedad Española para el Estudio del Patrimonio Histórico-Educativo (SEPHE), la Sociedad Española de Historia de la Educación (SEDHE), la red de Institutos históricos, los Museos Pedagógicos de Aragón y Galicia, el Centro Internacional de la Cultura Escolar (CEINCE) o universidades como las de Alcalá de Henares, Complutense de Madrid, Murcia, Palma, Salamanca, Sevilla, UNED, Valencia o Vic.

Los encuentros científicos son, probablemente, uno de los exponentes más significativos de la evolución experimentada tanto de los intereses generales de los investigadores como de los avances del conocimiento de la historia del patrimonio educativo en nuestro país a lo largo de la última década. Las primeras tentativas seminales que contribuyeron a gestar, impulsar, orientar y consolidar 
esta parcela historiográfica se debieron al Museo Pedagógico de Galicia, que organizaría el I Foro Ibérico de Museísmo Pedagóxico, en Santiago de Compostela, en 2001 (PEÑA SAAVEDRA, 2003), y a la Sociedad Española de Historia de la Educación (SEDHE), que dedicaría el XII Coloquio Nacional de Historia de la Educación, realizado en Burgos, en 2003, al estudio de la "Etnohistoria de la escuela". (COLOQUIO Nacional de Historia de la Educación, 2003).

Diferentes jornadas científicas de Historia de la Educación han dedicado puntualmente, a lo largo de estos años, entre otras instancias, alguna de sus ediciones a abordar diferentes vertientes del patrimonio educativo. Han sido los casos, por ejemplo, de las V Jornades d'Història de l'Educació Valenciana, celebradas en 2009, que centraron su atención en El Parimoni historicoeducatiu valencià (MAYORDOMO PÉREZ; AGULLÓ DÍAZ; GARCÍA FRASQUET, 2011) o las XXI Jornades d'Història de l'Educació, de la Societat d'Història de l'Educació dels Països de Llengua Catalana, realizadas en Palma, en 2014, tituladas Imatges de l'escola, imatge de l'educació. (COMAS RUBÍ et al., 2014).

Pero las iniciativas que han alcanzado un mayor grado de estabilidad y regularidad, aquellas que surgieron con la finalidad expresa y específica del estudio del patrimonio educativo, han sido promovidas por dos entidades. Por una parte, por la Asociación Nacional para la Defensa del patrimonio de los Institutos Históricos (ANDPIH), la cual ha celebrado con una periodicidad anual, desde 2007 a 2015, un total de nueve ediciones de las denominadas Jornadas de Institutos Históricos Españoles (Granada, 2007, San Cristóbal de la Laguna, 2008, Guadalajara, 2009, Santiago de Compostela, 2010, Cabra, 2011, Logroño, 2012, Burgos, 2013, Badajoz, 2014, Mahón, 2015). Unas reuniones que han permitido abordar, estudiar y reflexionar acerca de las singularidades de la salvaguarda, estudio, gestión y puesta en valor que requieren las colecciones bibliográficas, archivísticas y de material científico que, entre otras, atesoran las instituciones de enseñanza secundaria más longevas, aquellas que cuentan con más de ciento cincuenta años de existencia. Las actas de tales jornadas pueden consultarse a través de la URL de la Asociación (en: < https://sites.google.com/ site/andelpih/>). Por otra parte, la segunda entidad que ha desarrollado una actividad científica más prolífica y continuada en estas lides ha sido la Sociedad Española para el Estudio del Patrimonio Histórico-Educativo (SEPHE), la cual ha llevado a cabo, entre 2005 y 2014, seis jornadas cientificas. Unas jornadas abiertas dirigidas, no solo a quienes forman parte de dicha Sociedad sino a la comunidad científica en general, que han constituido una de las vías fundamentales para el avance del conocimiento sobre el patrimonio educativo en nuestro país, a las que le vamos a dedicar una atención especial.

La SEPHE ha venido encomendando la organización de sus jornadas científicas a diferentes entidades. Las I Jornadas que, corrieron a cargo del Museo 
Pedagógico de Galicia (MUPEGA), se celebraron en Santiago de Compostela, del 11 al 13 de mayo de 2005, y abordaron El museísmo pedagógico en España: actualidad y perspectivas, luces y sombras. (PEÑA SAAVEDRA, 2005). El Centro Internacional de la Cultura Escolar (CEINCE) se ocupó de organizar las II Jornadas, realizadas en Berlanga de Duero (Soria), del 9 al 12 de mayo de 2007, sobre el tema La cultura material de la escuela. En el centenario de la Junta de Ampliación de Estudios, 1907-2007. (ESCOLANO BENITO, 2007). El Museo Pedagógico de Aragón asumió la programación de las III Jornadas, efectuadas en Huesca, del 1 al 3 de octubre de 2008, que versaron sobre los Museos Pedagógicos y la memoria recuperada. (JUAN, 2008). Las IV Jornadas fueron coordinadas por el Museo Virtual de Pedagogía de la Universidad de Vic (MUVIP), en Vic (Barcelona), del 8 al 10 de septiembre de 2010, bajo el título La memoria educativa en los museos de educación y pedagogía como proyecto de ciudadanía. (COLLELLDEMONT; PADRÓS; CARRILLO, 2010). Las V Jornadas, que integrarían, al unísono, al III Foro Ibérico de Museísmo Pedagógico, las llevó a cabo el Centro de Estudios sobre la Memoria Educativa (CEME), de la Universidad de Murcia, en Murcia, del 21 al 23 de noviembre de 2012, centrando su atención sobre Patrimonio y Etnografía de la escuela en España y Portugal durante el siglo XX. (MORENO MARTÍNEZ; SEBASTIÁN VICENTE, 2012). Las VI Jornadas fueron coordinadas, conjuntamente, por tres universidades (la Universidad Complutense de Madrid, la Universidad Nacional de Educación a Distancia y la Universidad de Alcalá) y dos institutos históricos madrileños (el IES San Isidro y el IES Isabel la Católica), tuvieron lugar en Madrid del 22 al 24 de octubre de 2014, y llevaron por título Pedagogía Museística. Prácticas, usos didácticos e investigación del patrimonio educativo. (BADANELLI RUBIO et al., 2014).

Una aproximación a la evolución de las inscripciones con las que han contado estas jornadas evidencia su grado de consolidación y una tendencia patente hacia un progresivo incremento del número de participantes. De un número de 29 asistentes a las primeras jornadas se ha ido pasando, paulatinamente, a un centenar en las sextas. En las seis ediciones se ha contado con un total de 336 personas inscritas, de las que, 288 desempeñaban su actividad profesional en 25 universidades, seis museos y centros de estudios, cinco institutos históricos y otras entidades españolas. Asimismo, la progresiva internacionalización de las jornadas, especialmente reseñable en las dos últimas, ha supuesto la inscripción de 48 personas vinculadas a ocho universidades brasileñas, cinco portuguesas, cuatro italianas, dos belgas, una argentina y otra mejicana, así como a otras cuatro entidades portuguesas. 
CUADRO 1 - LAS APORTACIONES EFECTUADAS A LAS JORNADAS CIENTÍFICAS DE LA SOCIEDAD ESPAÑOLA PARA EL ESTUDIO DEL PATRIMONIO HISTÓRICO-EDUCATIVO (SEPHE) $(2005-2014)^{*}$

\begin{tabular}{|c|c|c|c|c|c|c|}
\hline $\begin{array}{l}\text { Tipología de bienes o temas de estudio sobre } \\
\text { patrimonio y museísmo pedagógico }\end{array}$ & $\begin{array}{c}\text { I } \\
\text { Jornadas }\end{array}$ & $\begin{array}{c}\text { II } \\
\text { Jornadas }\end{array}$ & $\begin{array}{c}\text { III } \\
\text { Jornadas }\end{array}$ & $\begin{array}{c}\text { IV } \\
\text { Jornadas }\end{array}$ & $\begin{array}{c}\mathrm{V} \\
\text { Jornadas }\end{array}$ & $\begin{array}{c}\text { VI } \\
\text { Jornadas }\end{array}$ \\
\hline \multicolumn{7}{|l|}{ 1. Bienes materiales } \\
\hline 0. Estudios generales sobre cultura material & & $4 * *$ & 1 & 2 & $2 * *$ & 4 \\
\hline 1. Manualística escolar & & 2 & 6 & & 3 & 3 \\
\hline 2. Bienes archivísticos & & 1 & & 1 & 4 & 8 \\
\hline 3. Bienes arquitectónicos & & & 1 & 1 & 9 & 5 \\
\hline \multicolumn{7}{|l|}{ 4. Bienes didácticos: } \\
\hline $\begin{array}{l}\text { 4.a) Científicos, tecnológicos y/o ciencias } \\
\text { naturales }\end{array}$ & & 2 & & 2 & 3 & 1 \\
\hline 4.b) Matemáticas y/o geometría & & 1 & 1 & 2 & 2 & 1 \\
\hline 4.c) Geografía & & & & & & 1 \\
\hline 4.d) Música & & & & & & 1 \\
\hline \multicolumn{7}{|l|}{ 4.e) Gimnástica } \\
\hline 4.f) Artísticos / trabajos manuales & & & & & & 1 \\
\hline 4.g) Lengua & & & & & 1 & \\
\hline 4.h) Historia & & & & & 1 & 1 \\
\hline 5. Bienes pedagógicos & & & & & & 1 \\
\hline 6. Objetos & & 1 & & & & 1 \\
\hline 7. Cuadernos escolares & & & & 1 & 1 & 1 \\
\hline 8. Fuentes & & & & & 3 & 4 \\
\hline Total bloque 1 & & 11 & 9 & 9 & 31 & 33 \\
\hline \multicolumn{7}{|l|}{ 2. Bienes inmateriales } \\
\hline $\begin{array}{l}\text { 0. Estudios generales sobre cultura } \\
\text { inmaterial }\end{array}$ & & & & & & 1 \\
\hline 1. La memoria individual del alumnado & & & 2 & & 3 & 1 \\
\hline 2. La memoria individual del profesorado & & & 4 & & 1 & 1 \\
\hline 3. Memoria colectiva e institucional & & & 3 & & 1 & 2 \\
\hline 4. La memoria depositada en otras fuentes & & & 5 & & 3 & \\
\hline Total bloque 2 & & & 14 & & 8 & 5 \\
\hline \multicolumn{7}{|l|}{ 3. Museísmo pedagógico } \\
\hline 0. Estudios generales & & 2 & & $5 * *$ & 1 & 3 \\
\hline 1. Centros museográficos & $6 * *$ & 4 & & & 2 & 3 \\
\hline 2. Museos virtuales & & 1 & & 2 & 1 & \\
\hline 3. Proyectos centros museográficos & 2 & 2 & & & 1 & \\
\hline 4. Proyectos museos virtuales & 1 & & & & & 2 \\
\hline 5. Experiencias & 5 & & 3 & 1 & 1 & 11 \\
\hline 6. Gestión de colecciones & & 1 & & & & 5 \\
\hline Total bloque 3 & 14 & 10 & 3 & 8 & 6 & 24 \\
\hline Total & 14 & 21 & 26 & 17 & 45 & 62 \\
\hline
\end{tabular}

* Elaboración propia a partir de los libros de actas. ** Incluye conferencia inaugural.

FUENTE: El autor (2015). 
Para efectuar una aproximación al balance general de las aportaciones realizadas hemos partido del análisis de los libros de actas de las jornadas computando las conferencias, ponencias y comunicaciones publicadas en las mismas. De ese modo podemos conocer la presencia y evolución de los temas objeto de estudio tratados. Con tal fin, como ya hiciéramos y detalláramos en un trabajo anterior (MORENO MARTÍNEZ, 2015), se han establecido tres bloques temáticos, referidos a bienes materiales, inmateriales y a museísmo pedagógico, partiendo para la primera modalidad de bienes mencionada de la tipología propuesta por Meda y Badanelli (2013, p. 171-173).

Como se observa en el Cuadro 1 de las 185 aportaciones publicadas, una de cada dos, noventa y tres en total, han abordado cuestiones relativas a la cultura material de las instituciones educativas. Los trabajos estaban referidos, fundamentalmente, al contexto español, pero también a otros países como, especialmente, Portugal, así como Italia, Brasil, Méjico o Uruguay. Los bienes arquitectónicos han sido bienes tangibles que han despertado mayor atención, con un número total de dieciséis trabajos. Colaboraciones que han estudiado, sobre todo, grupos escolares emblemáticos de diferentes ciudades españolas (A Coruña, Málaga, Madrid o Pontevedra), portuguesas (Penafiel) o mejicanas (México), espacios educativos singulares o sus representaciones. Asimismo, la manualística escolar, con catorce trabajos, ha sido, otra de las parcelas a la que los investigadores han prestado una atención destacada. En este apartado encontramos trabajos sobre bibliotecas escolares, la adquisición de valores por medio de los textos de diversas materias, manuales de diferentes disciplinas, libros de lectura, los manuales escolares en contextos nacionales determinados, como el uruguayo o el italiano, colecciones específicas, bases de datos o literatura infantil. Otras catorce aportaciones se han ocupado de diferentes vertientes de los bienes archivísticos, fondos especiales, su salvaguarda, gestión, sus potencialidades para la investigación y como recurso didáctico. Los estudios generales sobre la cultura material han contado con trece aportaciones acerca de las dimensiones antropológicas, históricas, historiográficas, etnográficas o identitarias del patrimonio educativo. Pero, como puede apreciarse, el mayor número de aportaciones efectuadas, con un total de veintiún trabajos, coincide con el apartado referido a los bienes didácticos. De ellos, dos ámbitos disciplinares parecen haber acaparado, particularmente, el interés principal de los estudios, como son las ciencias, la tecnología y las ciencias naturales, así como las matemáticas y/o la geometría.

El museísmo pedagógico ha sido, tras la cultura material de las instituciones educativas, con un total de 65 colaboraciones, la parcela temática que ha suscitado una mayor atención por parte de los participantes en las jornadas de la SEPHE. Un hecho comprensible teniendo en cuenta que, más allá de la importancia y la creciente vitalidad que tales iniciativas han experimentado en 
los últimos tiempos, algunas jornadas han centrado su atención en su estudio, en unos casos monográficamente, como fueron las I jornadas, o de forma destacada al contar con secciones expresamente consignadas al efecto, como sucedió en las II Jornadas, con una tercera sección titulada "Museología y museografía de la educación. Experiencias y proyectos", o en las V que, como se ha mencionado, constituyeron a un tiempo el III Foro Ibérico de Museísmo Pedagógico, cuya cuarta sección se dedicó a "La escuela reconstruida: pasado, presente y futuro del Museísmo pedagógico". El número más elevado de aportaciones, con un total de veintiuna, lo representan aquellos trabajos dedicados al análisis de experiencias relativas a la conservación, estudio, difusión, potencialidades educativas y usos didácticos del patrimonio histórico-educativo. Un tipo de trabajos muy frecuente, con quince aportaciones, engloba aquellas colaboraciones que se ocupan en dar a conocer los museos pedagógicos y otros centros de investigación afines. También han sido motivo de especial atención los estudios generales sobre diferentes vertientes de la museología o la museografía pedagógica, su relación con la historia de la educación, los proyectos de creación de nuevos espacios museísticos, las iniciativas asociadas a museos virtuales o las posibilidades y acciones emprendidas a través de internet.

El tercer bloque de contenidos abordados en el transcurso de las jornadas hacía referencia a los bienes inmateriales, a la memoria individual, las vivencias y los recuerdos escolares de alumnos y docentes, a la memoria colectiva de grupos e instituciones, así como a otras manifestaciones o representaciones de la memoria. El número total de colaboraciones relacionadas con estos temas, sensiblemente inferior a las de los dos bloques anteriores, ascendió a veintisiete comunicaciones. En cuanto al contenido de las mismas, seis tuvieron por objeto el estudio de diferentes vertientes de la memoria de los escolares y otras tantas del profesorado. La memoria colectiva y/o institucional también estuvo presente en siete colaboraciones que estudiaban iniciativas de colectivos, centros formativos e instituciones académicas de diferentes niveles educativos. Otros ocho trabajos se caracterizaron por recurrir a fuentes singulares para reconstruir diferentes facetas de la cultura de las instituciones educativas, tales como publicaciones periódicas, prensa escolar, revistas profesionales, prensa general, inventarios escolares, los nombres de los centros educativos o la fotografía.

\section{Conclusiones}

La historiografía del patrimonio educativo en España ha experimentado, a lo largo de los últimos quince años, un avance reseñable. A ello han contribuido 
diversos factores. Entre ellos cabe destacar la función desempeñada por las exposiciones pedagógicas que abarcando un amplio espectro temático, y aún estando pendiente de un análisis más profundo y pormenorizado de las mismas, puede señalarse que para la historiografía del patrimonio han contribuido por una parte a favorecer e impulsar la investigación, la recuperación y reconstrucción de la memoria y la historia de la educación y de su patrimonio y, por otra, a difundir y visibilizar los resultados de la investigación desarrollada. Asimismo, la creciente recuperación e impulso del museísmo pedagógico tampoco ha sido ajeno a los avances de la investigación sobre el patrimonio. La estrecha vinculación producida entre cierto tipo de entidades museísticas, grupos de investigación universitarios, proyectos de investigación y jornadas científicas ha convertido a algunas de estas iniciativas en parte de las consecuencias de dicha labor investigadora, así como en nuevas plataformas para afrontar nuevos retos para la investigación del patrimonio educativo. La promoción y consolidación alcanzada por los encuentros científicos son un claro exponente de la vitalidad adquirida, en poco más de una década, por la investigación histórica del patrimonio educativo. Según evidencian las jornadas científicas organizadas por la Sociedad Española para el Estudio del Patrimonio Educativo (SEPHE), entre 2005 y 2014, la cultura material de las instituciones educativas y el museísmo pedagógico constituyen las líneas de investigación sobre el patrimonio educativo dominantes en nuestro contexto.

\section{REFERENCIAS}

BADANELLI RUBIO, A. M.; POVEDA SANZ, M.; RODRÍGUEZ GUERRERO, C. (Coords.). Pedagogía Museística. Prácticas, usos didácticos e investigación del patrimonio educativo. Madrid: Universidad Complutense de Madrid, 2014.

BOLETÍN INFORMATIVO SEPHE. Sociedad Española para el Estudio del Patrimonio Histórico-Educativo. 2006-2014. Disponible en: $<$ http://institucional.us.es/paginasephe/ actividades.html >. Acceso en: 29 mayo 2015.

COLLELLDEMONT, E.; PADRÓS, N.; CARRILLO, I. (Coords.). Memoria, ciudadanía y museos de educación. Vic: Universitat de Vic, 2010.

COLOQUIO Nacional de Historia de la Educación. Etnohistoria de la escuela. XII Coloquio Nacional de Historia de la Educación. Burgos, 18-21 junio 2003. Burgos: Servicio de Publicaciones de la Universidad de Burgos, 2003.

COMAS RUBÍ, F.; GONZÁLEZ GÓMEZ, S.; MOTILLA SALAS, X.; SUREDA GARCIA, B. (Eds.). Imatges de l'escola, imatge de l'educació. Actes de les XXI Jornades 
d'Història de l'Educació. Palma, del 26 al 28 de novembre de 2014. Palma: Universitat de les Illes Balears, 2014.

CAMPILLO FLORES, I.; COLLELDEMONT PUJADAS, E. Construir un museo pedagógico virtual. Fundamentos teóricos y elementos de gestión. En: ESCOLANO BENITO, A. (Ed.). La cultura material de la escuela. En el centenario de la Junta de Ampliación de Estudios, 1907-2007. Berlanga de Duero: CEINCE, 2007.

ESCOLANO BENITO, A. (Ed.). La cultura material de la escuela. En el centenario de la Junta de Ampliación de Estudios, 1907-2007. Berlanga de Duero: CEINCE, 2007.

GARCÍA DEL DUJO, A. Museo Pedagógico Nacional (1882-1941). Salamanca: Ediciones de la Universidad de Salamanca, 1985.

HERNÁNDEZ DÍAZ, J. M. Las exposiciones pedagógicas y la historia material de la educación. En: Educación y europeísmo. De Vives a Comenio. Málaga: Universidad de Málaga-Sociedad Española de Historia de la Educación, 1993.

HERNÁNDEZ DÍAZ, J. M. Museos pedagógicos y exposiciones educativas en España en los inicios del siglo XXI. En: PEÑA SAAVEDRA, V. (Coord.). I Foro Ibérico de Museísmo Pedagógico. O museísmo pedagógico en España e Portugal: itinerarios, experiencias e perspectivas. Actas. Santiago de Compostela: Xunta de Galicia, 2003.

JUAN, V. (Ed.). Museos Pedagógicos. La memoria recuperada. Huesca: Museo Pedagógico de Aragón, 2008.

JULIA, D. La culture scolaire comme objet historique. En: Colonial Experience in Education. Historical Issues and Perspectives. Gante: Paedagogica Historica, Supplementary series (I), 1995.

LAWN, M. Modelling the Future: exhibitions and materiality of education. Oxford: Symposium Books, 2009.

MAYORDOMO PÉREZ, A.; AGULlÓ DÍAZ, M. C.; GARCÍA FRASQUET, G. (Coords.). El Parimoni histórico educatiu valencià. V Jornades d'Història de l'Educació Valenciana. Gandia, 30 i 31 d'octubre de 2009. Gandia: CEIC Alfons el Vell, 2011.

MEDA, J.; BADANELLI, A. M. (Eds.). La historia de la cultura escolar en Italia y en España: balance y perspectivas. Macerata: Edizioni Università di Macerata (EUM), 2013.

MORENO MARTÍNEZ, P. L. Las Jornadas Científicas de la Sociedad Española para el Estudio del Patrimonio Histórico-Educativo (SEPHE) y el avance del conocimiento (2005-2012). En: COLMENAR ORZAES, C.; RABAZAS ROMERO, T. (Eds.). Educación e investigación. El legado pedagógico de Julio Ruiz Berrio. Madrid: Biblioteca Nueva, 2015.

MORENO MARTÍNEZ, P. L.; SEBASTIÁN VICENTE, A. (Eds.). Patrimonio y Etnografía de la escuela en España y Portugal durante el siglo XX. Murcia: Sociedad Española para el Estudio del Patrimonio Histórico-Educativo y Centro de Estudios sobre la Memoria Educativa (CEME) de la Universidad de Murcia, 2012. 
OTERO URTAZA, E. El viaje de Manuel Bartolomé Cossío por los Museos Pedagógicos de Europa en 1882. En: I Encontro Iberoamericano de Museos Pedagóxicos e Museólogos da Educación. Santiago de Compostela, 20 a 22 de febrero de 2008. Santiago de Compostela: Museo Pedagóxico de Galicia, 2008.

PEÑA SAAVEDRA, V. (Coord.). I Foro Ibérico de Museísmo Pedagóxico. O museísmo pedagóxico en España e Portugal: itinerarios, experiencias e perspectivas. Actas 2001. Museo Pedagóxico de Galicia: Santiago de Compostela, 2003.

PEÑA SAAVEDRA, V. (Dir.). I Jornadas Cientificas de la Sociedad Española para el Estudio del Patrimonio Histórico-Educativo. El museísmo pedagógico en España: actualidad y perspectivas, luces y sombras. Actas, documentos y testimonios. Santiago de Compostela: Xunta de Galicia, 2005.

POZO ANDRÉS, M. M. del. The Bull and the Book: images of Spain and Spanish education in the World Fairs of the nineteenth century, 1851-1900. En: LAWN, M. Modelling the Future: exhibitions and materiality of education. Oxford: Symposium Books, 2009.

RUIZ BERRIO, J. (Ed.). El patrimonio histórico-educativo. Su conservación y estudio. Madrid: Biblioteca Nueva, 2010.

Texto recebido em 11 de outubro de 2015. Texto aprovado em 11 de outubro de 2015. 